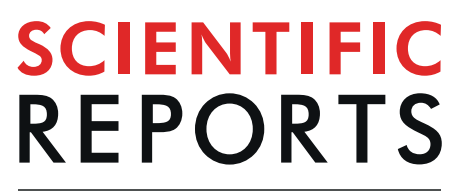

natureresearch

\title{
Discovery of a new Theileria sp. closely related to Theileria annulata in cattle from Sri Lanka
}

Thillaiampalam Sivakumar ${ }^{1}$, Shiori Fujita ${ }^{1}$, Bumduuren Tuvshintulga $\mathbb{1}^{1}$,
Hemal Kothalawala ${ }^{2}$, Seekkuge Susil Priyantha Silva $^{2}$ \& NaoakiYokoyama ${ }^{1,3^{*}}$

Theileria annulata is a haemoprotozoan parasite that causes a cancer-like illness known as tropical theileriosis in cattle. In the course of analyzing the genetic diversity of $T$. annulata in Sri Lanka, we observed that merozoite-piroplasm surface antigen (tams1) and surface protein (tasp)-like gene sequences obtained from bovine blood DNA samples, which were PCR-positive for T. annulata, were conserved but shared low identity with $T$. annulata GenBank sequences. Moreover, the 18S rRNA sequences from the Sri Lankan samples contained ten unique single-nucleotide polymorphisms compared with all known $T$. annulata sequences. The cytochrome b (cob) gene sequences isolated from the Sri Lankan samples were highly conserved and shared low identity scores with similarly conserved T. annulata sequences from GenBank. Phylogenetic analysis showed that the Sri Lankan tams1-like, tasp-like, 18S rRNA, and cob sequences clustered together and formed sister clades to the common ancestors of all known $T$. annulata and Theileria lestoquardi sequences. These findings demonstrated that the Sri Lankan cattle were not infected with $T$. annulata but with a new Theileria sp. (designated as Theileria sp. Yokoyama) closely related to $T$. annulata.

The Theileria genus consists of tick-borne haemoprotozoan parasites of ruminants ${ }^{1}$. Among them, Theileria annulata infects cattle and buffalo, and causes a lymphoproliferative disease known as tropical theileriosis ${ }^{2}$. The disease is usually characterised by enlarged lymph nodes, fever, increased pulse and respiratory rates, swollen eyelids, profuse lachrymation, anaemia, jaundice, and sometimes death ${ }^{3,4}$. Therefore, tropical theileriosis is considered an economically significant disease in endemic countries ${ }^{5-7}$. Long-term survival of T. annulata in host animals is facilitated by the protozoan's genetic diversity, which helps the parasite escape the host's immune response $^{8,9}$. Therefore, T. annulata's genetic diversity has been analysed in several endemic countries based on genes that encode surface proteins, such as the Theileria annulata merozoite-piroplasm surface antigen (tams1) and Theileria annulata surface protein (tasp) genes ${ }^{10-13}$. The tams 1 and tasp genes have been demonstrated to be highly diverse in most endemic countries investigated to date.

Sri Lanka is a tropical country, and previous PCR-based studies found that infection with T. annulata is common among the country's cattle populations ${ }^{14,15}$. However, the genetic diversity of T. annulata remains to be investigated in Sri Lanka. Therefore, in the present study, we analysed the tams 1 and tasp gene sequences in DNA samples from Sri Lankan bovine blood, which were PCR-positive for T. annulata. As the findings suggested that the genetic backgrounds of Sri Lankan isolates might be different from those of T. annulata, further analyses were conducted using the $18 \mathrm{~S}$ rRNA and cytochrome $\mathrm{b}(c o b)$ gene sequences.

\section{Results}

Thirty-nine bovine blood DNA samples from the Polonnaruwa and Nuwara Eliya districts in Sri Lanka, which had tested positive on a T. annulata-specific PCR assay, were used in the present study ${ }^{15}$. From these samples, tams1-like gene sequences were successfully isolated from 34 samples (GenBank accession no. LC467536 LC467569), including 28 from Polonnaruwa and 6 from Nuwara Eliya. The newly determined tams1-like nucleotide and translated amino acid sequences were highly conserved but shared low identity scores with $T$. annulata sequences $(n=125)$ from ten countries: Sudan, Tunisia, Mauritania, Bahrain, Turkey, Italy, Spain, Portugal,

\footnotetext{
${ }^{1}$ National Research Center for Protozoan Diseases, Obihiro University of Agriculture and Veterinary Medicine, Hokkaido, Japan. ${ }^{2}$ Veterinary Research Institute, Peradeniya, Sri Lanka. ${ }^{3}$ OIE reference laboratory for bovine babesiosis and equine piroplasmosis, National Research Center for Protozoan Diseases, Obihiro University of Agriculture and Veterinary Medicine, Hokkaido, Japan. *email: yokoyama@obihiro.ac.jp
} 


\begin{tabular}{|l|l|l|l|l|l|l|}
\hline \multirow{2}{*}{ Gene } & \multicolumn{4}{l}{ Nucleotide sequences } & \multicolumn{2}{l|}{ Amino acid sequences } \\
\cline { 2 - 7 } & Sri Lankan & T. annulata & $\begin{array}{l}\text { Between Sri Lankan } \\
\text { and } \text { T. annulata }\end{array}$ & Sri Lankan & T. annulata & $\begin{array}{l}\text { Between Sri Lankan } \\
\text { and } \text { T. annulata }\end{array}$ \\
\hline tams1 & $98.9-100$ & $87.5-100$ & $87.2-93.4$ & $96.2-100$ & $80.4-100$ & $78.9-89.5$ \\
\hline tasp & $97.3-100$ & $85.4-100$ & $77.9-83.2$ & $95.4-100$ & $78.2-100$ & $67.5-73.9$ \\
\hline 18S rRNA (short) & $99.5-100$ & $99.1-100$ & $98.5-99.2$ & - & - & - \\
\hline 18S rRNA (long) & $99.7-100$ & $99.2-100$ & $98.7-99.3$ & - & - & - \\
\hline cob & $99.4-100$ & $98.5-100$ & $87.2-88.0$ & $98.8-100$ & $97.1-100$ & $82.5-84.8$ \\
\hline
\end{tabular}

Table 1. Nucleotide and amino acid identity scores (\%) shared among the Sri Lankan sequences, among the T. annulata sequences, and between the T. annulata and Sri Lankan sequences.

India, and China (Table 1). Multiple alignment of nucleotide sequences revealed that the TCC nucleotides, which were conserved across all T. annulata sequences at positions 598-600, were deleted in the Sri Lankan sequences (Fig. S1). The deletion of these nucleotides resulted in deletion of amino acid serine in Sri Lankan sequences (Fig. S2). Phylogenetically, the tams1 nucleotide and amino acid sequences from GenBank formed multiple clades, and the clade formed by the tams 1 homologous sequences in Theileria lestoquardi was nested within the T. annulata clades (Figs 1 and S3). However, the Sri Lankan sequences formed a sister clade to the common ancestor of all T. annulata and T. lestoquardi sequences in GenBank (Figs 1 and S3).

The tasp gene sequences were isolated from 32 DNA samples (GenBank accession no. LC467570 - LC467601), including 25 and 7 sequences from Polonnaruwa and Nuwara Eliya, respectively. Length polymorphism was observed among the newly determined sequences, with 22 and 10 sequences containing 1,023 bp and 1,020 bp, respectively. As previously reported, the tasp-like sequences had two short introns ${ }^{12}$, which were removed from the primer-trimmed Sri Lankan sequences to obtain 839- and 842-bp coding sequences. The Sri Lankan coding nucleotide and amino acid sequences shared $97.3-100 \%$ and $95.4-100 \%$ identity scores, respectively, with each other and $77.9-83.2 \%$ and $67.5-73.9 \%$ identity scores, respectively, with the $T$. annulata sequences $(\mathrm{n}=14)$ from Tunisia, Morocco, Turkey, India, and China (Table 1). The nucleotide and amino acid sequences at both ends of the tasp gene are usually conserved ${ }^{12}$. In contrast, the sequences in these regions, especially at the $5^{\prime}$ end in nucleotide sequences and $\mathrm{N}$-terminal region in amino acid sequences, were poorly conserved between the Sri Lankan and T. annulata sequences (Figs S4 and S5). Phylogenetic analysis showed that that T. lestoquardi surface protein gene sequences formed a sister clade to the tasp sequences from GenBank, while all the Sri Lankan sequences formed a sister clade to the common ancestor of clades formed by the T. annulata and T. lestoquardi gene sequences (Fig. 2). A phylogeny constructed based on translated amino acid sequences had the same topology (Fig. S6). These findings suggested that the Sri Lankan isolates have genetic backgrounds that differed from those of T. annulata.

However, although the tams 1 and tasp genes are markers for analysing genetic diversity among T. annulata, these genes are unsuitable for evolutionary studies because they evolved under immune pressure. Therefore, we isolated 1,185-bp 18S rRNA fragment (1,130 bp after primer regions were trimmed) from 37 DNA samples (GenBank accession no. LC467602 - LC467638), including 31 from Polonnaruwa and 6 from Nuwara Eliya. These $18 \mathrm{~S}$ rRNA sequences were highly conserved (99.5\%-100\% identities) and shared marginally low identities (98.0\%-99.2\%) with 63 T. annulata sequences from six countries, including Iran, Turkey, Spain, India, China, and Pakistan (Table 1). Phylogenetic analysis showed that the Sri Lankan sequences formed a monophyletic sister clade to the common ancestors of the T. annulata and T. lestoquardi $18 \mathrm{~S}$ rRNA sequences (Fig. 3). However, the clades were supported with low bootstrap values. Thus, we isolated relatively long fragment $(1,536 \mathrm{bp}$ after primer regions were trimmed) of $18 \mathrm{~S}$ rRNA sequences (GenBank accession no. LC495907 - LC495915), using a set of universal forward and reverse primers, from nine samples with single infection. In these sequences, we identified ten unique single-nucleotide polymorphisms (SNPs), including a deletion and nine substitutions compared with the GenBank-derived T. annulata sequences (Fig. S7). The topologies of phylogenetic trees constructed with short and long $18 \mathrm{~S}$ rRNA sequences were similar, but the bootstrap support for clades drastically increased in the later (Fig. 4).

Although the phylogenetic position and unique SNPs may identify the Sri Lankan isolates as a new Theileria sp., this assumption remains inconclusive as the $18 \mathrm{~S}$ rRNA sequences shared high identity scores with the T. annulata sequences. Therefore, we obtained $30 \mathrm{cob}$ gene sequences (GenBank accession no. LC467639 - LC467668), including 28 from Polonnaruwa and 2 from Nuwara Eliya, and compared them with the 43 T. annulata sequences from Sudan, Tunisia, Egypt, Turkey, India, and China. The T. annulata cob nucleotide and translated amino acid sequences from these countries were highly conserved, sharing $98.5-100 \%$ and $97.1-100 \%$ identity scores, respectively (Table 1). In contrast, these sequences shared only $87.2-88.0 \%$ and $82.5-84.8 \%$ identities with the Sri Lankan cob nucleotide and amino acid sequences, respectively (Figs S8 and S9). In addition, the Sri Lankan cob gene sequences were characterized by 122 unique SNPs compared with the GenBank-derived T. annulata sequences (Fig. S8). Phylogenetic analysis showed that the Sri Lankan cob nucleotide as well as amino acid sequences clustered together and formed a sister clade to the T. annulata clade (Figs 5 and S10), further supporting our assumption that the Theileria sp. from the cattle in Sri Lanka is not T. annulata but a Theileria sp. closely related to T. annulata. 


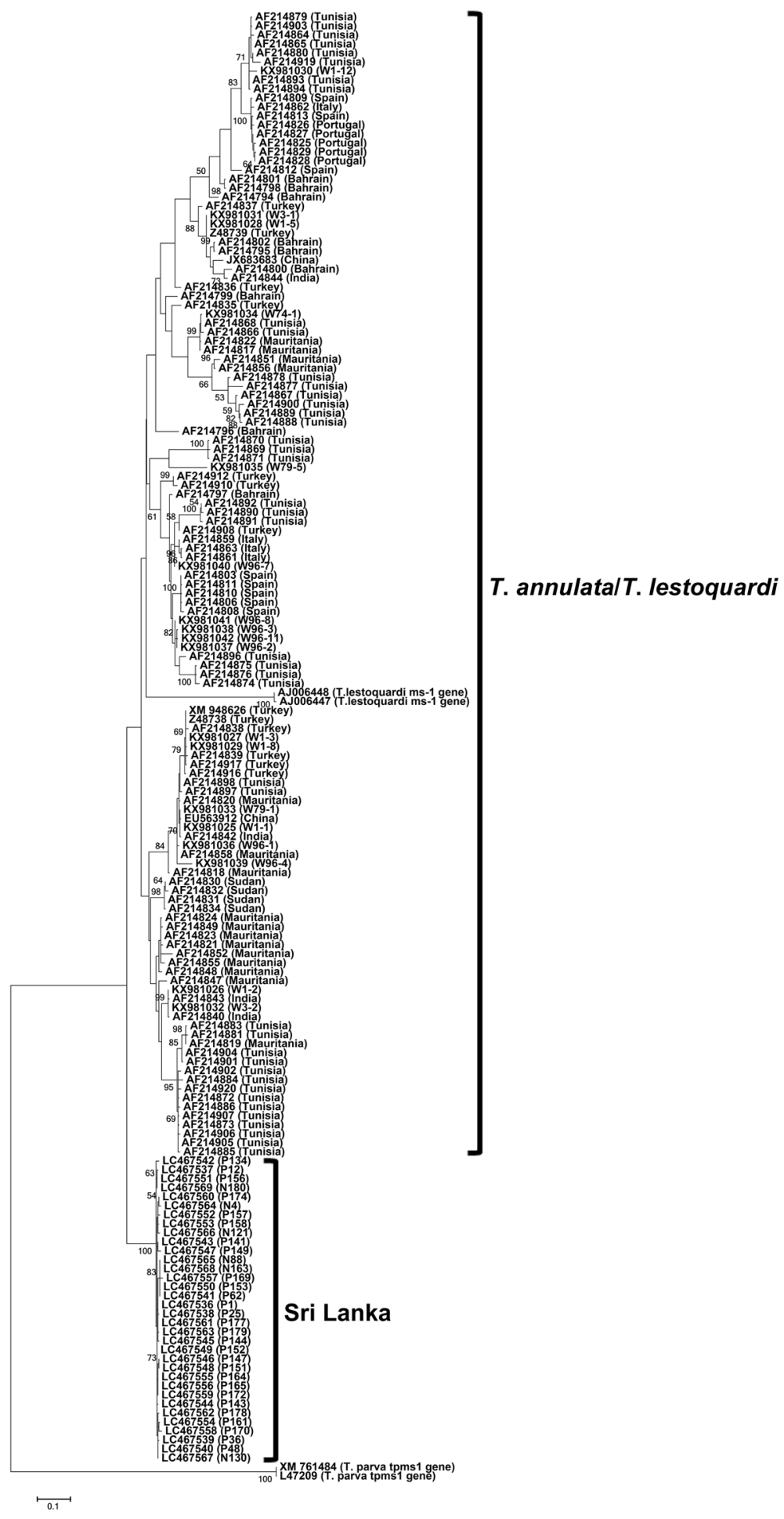

Figure 1. Phylogeny of the tams1 gene. A maximum-likelihood phylogenetic tree was constructed using 34 tams1-like gene sequences determined in the present study. The T. annulata and T. lestoquardi sequences were retrieved from GenBank. P and N series numbers provided with the Sri Lankan sequences indicate animal IDs from Polonnaruwa and Nuwara Eliya, respectively. The Sri Lankan sequences clustered together and formed a sister clade to the common ancestor of the clades formed by the T. annulata and T. lestoquardi sequences from other countries.

\section{Discussion}

The unique polymorphisms, low identity scores shared with the T. annulata sequences, and distinct phylogenetic positions collectively indicated that the parasite species from Sri Lanka, identified via PCR as T. annulata, was indeed a new Theileria sp. closely related to T. annulata. 


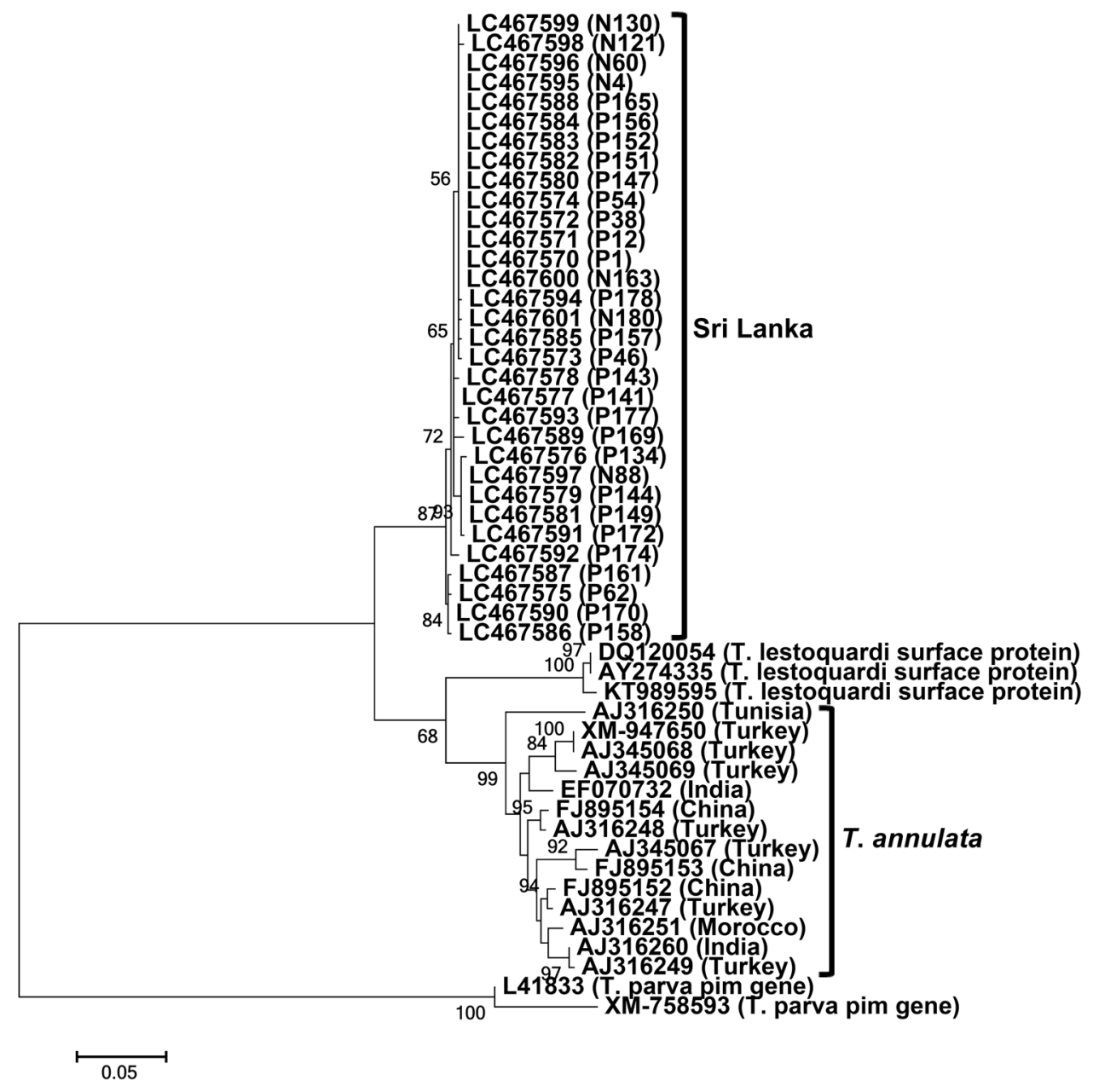

Figure 2. Phylogeny of the tasp gene. A maximum-likelihood phylogenetic tree was constructed using the coding sequences of 32 tasp-like gene sequences determined in the present study. The T. annulata and T. lestoquardi sequences were obtained from GenBank. P and N series numbers provided with the Sri Lankan sequences indicate animal IDs from Polonnaruwa and Nuwara Eliya, respectively. The Sri Lankan sequences clustered together and formed a sister clade to the common ancestor of the T. lestoquardi and T. annulata sequences from other countries.

Although the present study was initiated to analyse the genetic diversity of T. annulata in Sri Lanka, the tams1and tasp-like sequences from the Sri Lankan samples diverged from known T. annulata sequences. However, there is no bootstrap support for the T. annulata clade in tams 1 phylogeny. The genetic diversity of tams 1 is thought to be due to random intragenic recombination, which results in a mosaic pattern of diversity ${ }^{16}$. This could explain the lack of bootstrap support for the T. annulata clade. In addition, there are no bootstrap values for the separation of Sri Lankan clades in both tams 1 and tasp phylogenies due to lack of additional outgroup sequences. In tams 1 phylogeny, addition of further outgroup sequences, such as those from Theileria taurotragi and Theileria orientalis, however resulted in altered topology due to deletion of regions shared between T. annulata and T. lestoquardi from the alignment during phylogeny construction. Therefore, we refrained from using additional outgroup sequences in the tams 1 phylogeny. For the tasp phylogeny, we could not find suitable additional outgroup sequences.

Next, short $18 \mathrm{~S}$ rRNA sequences from the Sri Lankan samples were analysed. In phylogeny, the Sri Lankan sequences occurred in a clade unrelated to T. annulata. However, T. annulata, T. lestoquardi, and Sri Lanka clades and their separation were not supported by bootstrap values. Phylogenies constructed with short $18 \mathrm{~S}$ rRNA sequences are sometimes characterized by low bootstrap values, which usually improve when longer sequences are used ${ }^{17}$. Therefore, we isolated relatively longer $18 \mathrm{~S}$ rRNA sequences and used them for phylogeny construction. The topology of this phylogeny was similar to that constructed with short $18 \mathrm{~S}$ rRNA sequences, but the bootstrap support for each clade and their separation drastically improved. Theileria lestoquardi is thought to have recently evolved from T. annulata, following a host jump from cattle/buffalo to sheep/goat ${ }^{18}$. Despite the similarities between T. lestoquardi and T. annulata, they are considered as distinct parasite species because of the stringent host-specificity of the former as confirmed by in vitro and in vivo experiments ${ }^{19}$. Therefore, our observation that the Sri Lankan clade in $18 \mathrm{~S}$ rRNA phylogeny formed sister clade to the common ancestor of T. lestoquardi and T. annulata indicated that the Sri Lankan samples were infected with a new Theileria sp. 


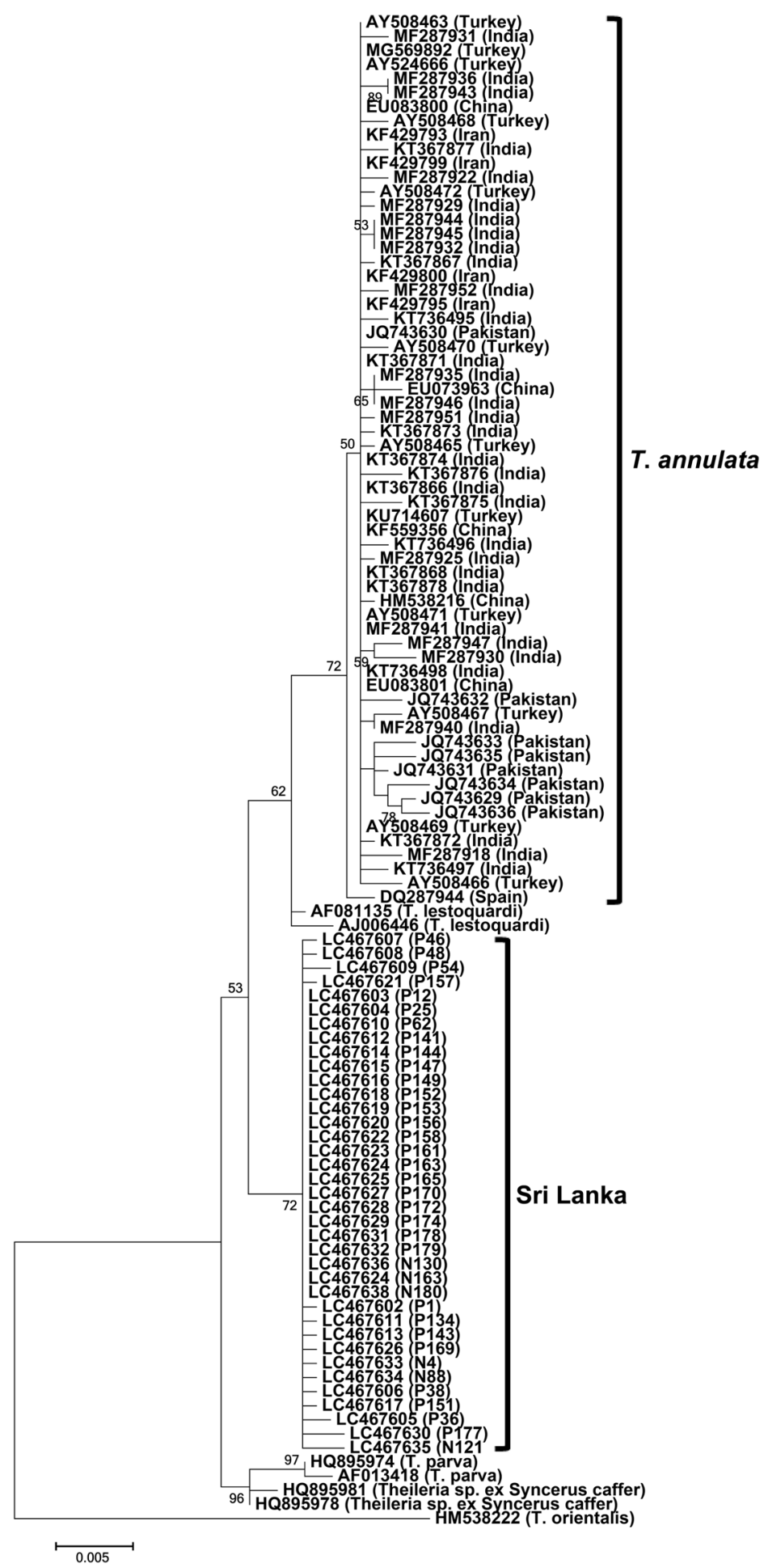

Figure 3. $18 \mathrm{~S}$ rRNA phylogeny. A maximum-likelihood phylogenetic tree was constructed using 37 short $18 \mathrm{~S}$ rRNA gene sequences $(1,130 \mathrm{bp})$ from Sri Lanka and the T. annulata and T. lestoquardi sequences from GenBank. P and N series numbers provided with the Sri Lankan sequences indicate animal IDs from Polonnaruwa and Nuwara Eliya, respectively. The Sri Lankan sequences clustered together and formed a sister clade to the common ancestor of the T. lestoquardi and T. annulata sequences from other countries.

However, in terms of identity scores, the Sri Lankan $18 \mathrm{~S}$ rRNA sequences differed only marginally from the T. annulata sequences. High identities are common between $18 \mathrm{~S}$ rRNA sequences from different Theileria species possibly due to recent speciation events ${ }^{20}$. The hypervariable region of the $18 \mathrm{~S}$ rRNA from T. lestoquardi differs by only two nucleotides compared with $T$. annulat ${ }^{21}$. Similarly, $18 \mathrm{~S}$ rRNA is highly conserved between Theileria 


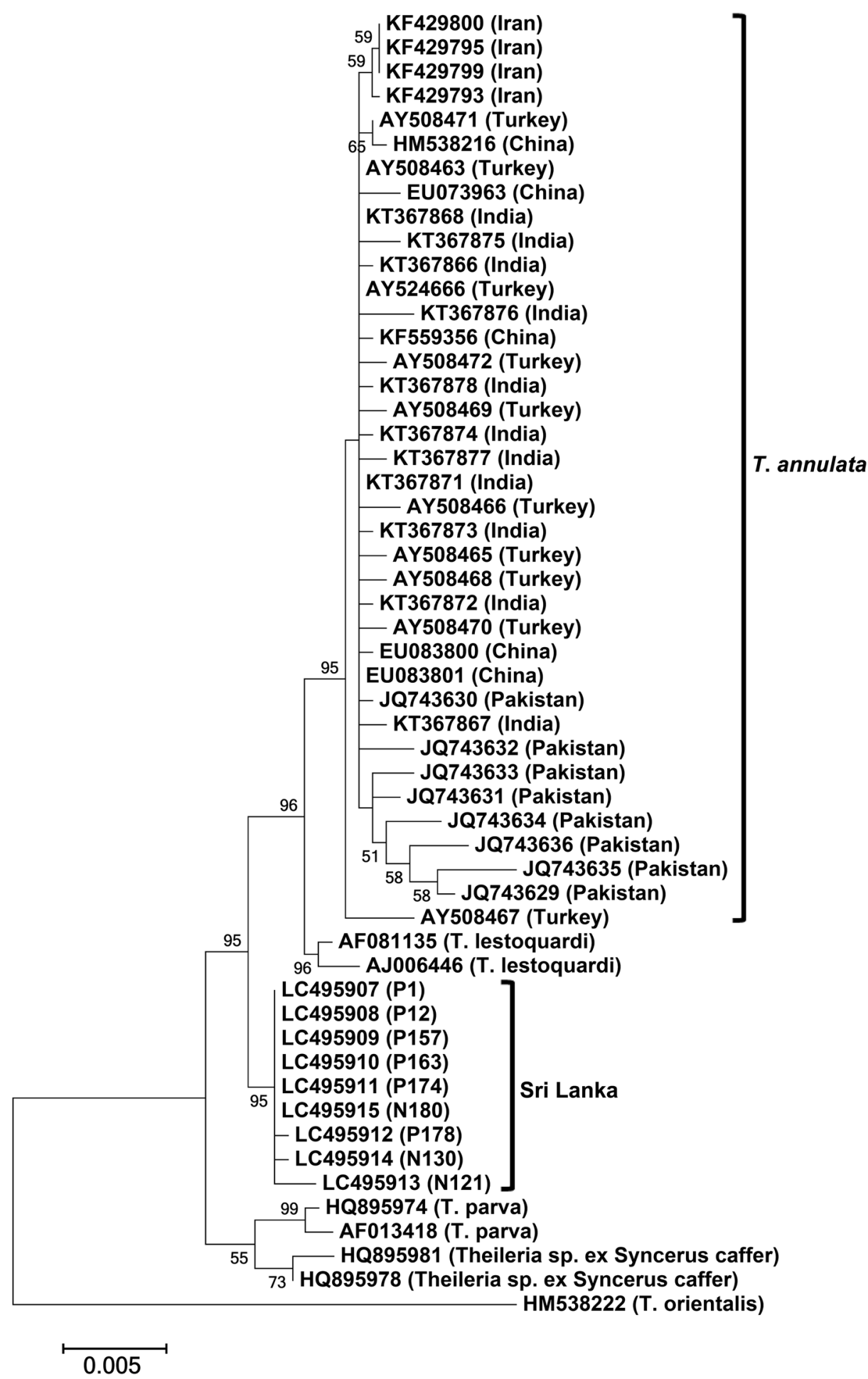

Figure 4. $18 \mathrm{~S}$ rRNA phylogeny. A maximum-likelihood phylogenetic tree was constructed using nine long $18 \mathrm{~S}$ rRNA gene sequences (1,536 bp) from Sri Lanka and the T. annulata and T. lestoquardi sequences from GenBank. P and N series numbers provided with the Sri Lankan sequences indicate animal IDs from Polonnaruwa and Nuwara Eliya, respectively. The Sri Lankan sequences clustered together and formed a sister clade to the common ancestor of the T. lestoquardi and T. annulata sequences from other countries. Note that the bootstrap support for T. annulata, T. lestoquardi, and Sri Lankan clades and their separation improved compared with that in phylogeny constructed with short $18 \mathrm{~S}$ rRNA sequences.

parva and Theileria sp. (buffalo) ${ }^{22}$. However, mitochondrial gene sequences adequately discriminated these parasite species ${ }^{20}$. Among the T. annulata mitochondrial genes, only the cob sequences were available in sufficient numbers in the GenBank. Therefore, we obtained the cob sequences from the Sri Lankan samples and compared 


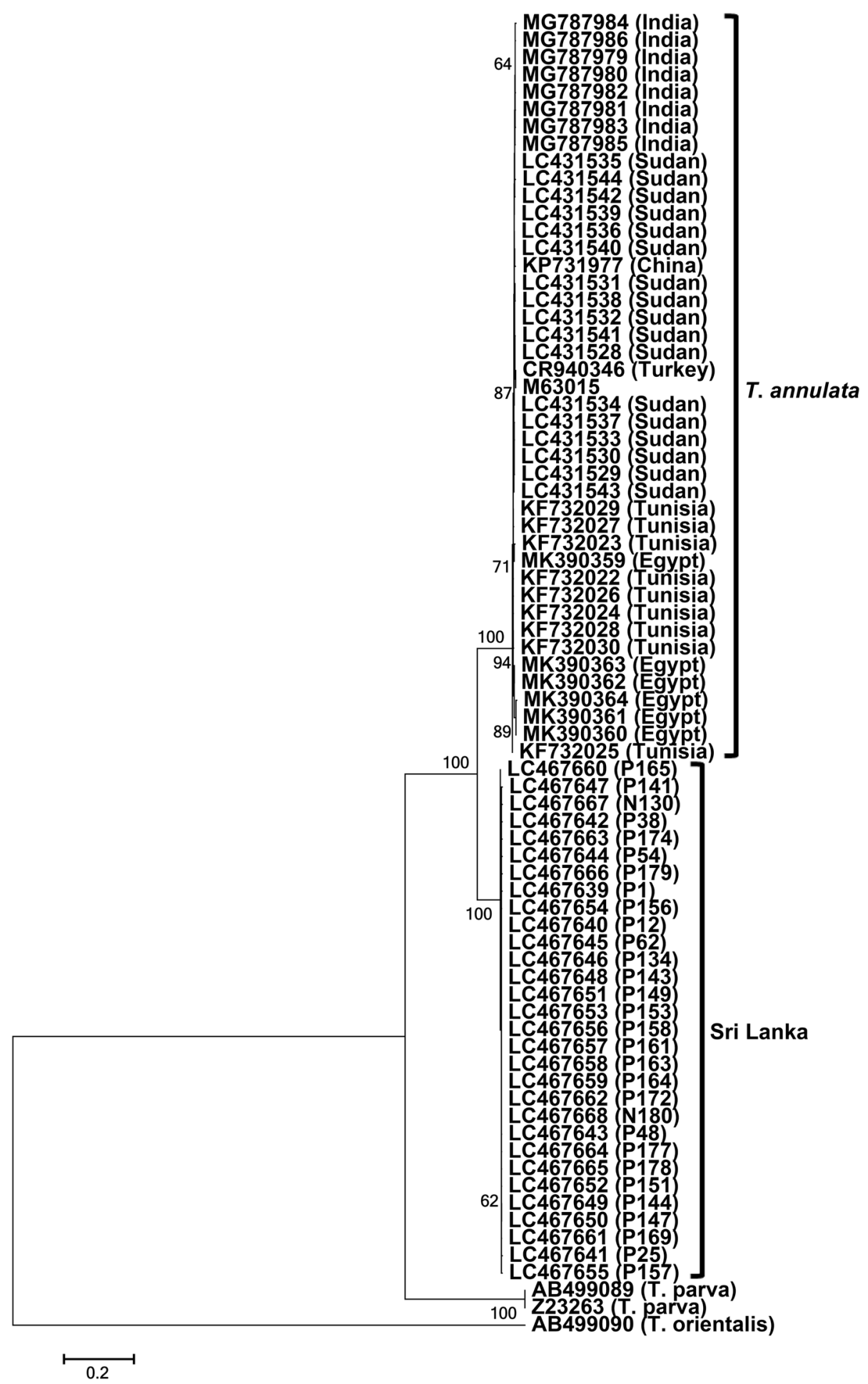

Figure 5. Phylogeny of the cob gene. A maximum-likelihood phylogenetic tree was constructed using $30 \mathrm{cob}$ gene sequences from Sri Lanka and the T. annulata sequences from GenBank. P and N series numbers provided with the Sri Lankan sequences indicate animal IDs from Polonnaruwa and Nuwara Eliya, respectively. The Sri Lankan sequences clustered together and formed a sister clade to the T. annulata sequences from other countries.

them with those from T. annulata isolates in different countries ${ }^{23,24}$. The Sri Lankan sequences were conserved but highly diverse compared with the similarly conserved T. annulata sequences. To the best of our knowledge, such a high diversity of mitochondrial genes was never reported for any species of protozoan parasites. These observations suggest that the parasite species, which had been previously identified as T. annulata in Sri Lanka, is a novel Theileria sp. 


\begin{tabular}{|c|c|c|c|c|c|}
\hline \multirow[b]{2}{*}{ Gene } & \multicolumn{2}{|l|}{ Primer sequence $\left(5^{\prime}-3^{\prime}\right)$} & \multirow{2}{*}{$\begin{array}{l}\text { Annealing } \mathrm{T} \\
\left({ }^{\circ} \mathrm{C}\right)\end{array}$} & \multirow{2}{*}{$\begin{array}{l}\text { Amplicon } \\
\text { size (bp) }\end{array}$} & \multirow{2}{*}{$\begin{array}{l}\text { Size (bp) after } \\
\text { primers were } \\
\text { removed }\end{array}$} \\
\hline & Forward & Reverse & & & \\
\hline tams1 & ATGTTGTCCAGGACCACCCTC & TTAAAGGAAGTAAAGGACTGATGAGAAG & 56 & 843 & 794 \\
\hline tasp & ATGAAATTCTTCTACCTTTTTGTTCTATTTCC & TTAACAACAATCTTCGTTAATGCGAG & 65 & 1020 and 1023 & 962 and 965 \\
\hline 18S rRNA (short) & AGGGCTAATACATGTTCGAGGCCATTT & TCCCTCTAAGAAGCGATAACGGGACAGT & 64 & 1185 & 1130 \\
\hline 18S rRNA (long) & AAGCCATGCATGTCTAAGTATAAGCTTTT & GAATAATTCACCGGATCACTCG & 56 & 1587 & 1536 \\
\hline$c o b$ & ATGAATTTGTTTAACTCACATTTGC & TTATGCACGAACTCTTGCAGAG & 55 & 1092 & 1045 \\
\hline
\end{tabular}

Table 2. List of primers, annealing temperatures, and sequence sizes. Primers designed in the present study were used to amplify tams 1, tasp, short fragment of $18 \mathrm{~S}$ rRNA, and cob genes, while long fragment of $18 \mathrm{~S}$ rRNA was amplified using previously described forward ${ }^{28}$ and reverse $\mathrm{e}^{29}$ primers.

The tams1-like gene sequences in the new Theileria sp. were highly conserved. Similarly, although the tasp-like sequences from Sri Lanka had some diversity, the gene was relatively conserved compared with the T. annulata sequences. Low genetic diversity among Theileria parasites is thought to be due to their short evolutionary histories. For example, the genetic diversity of $T$. parva in buffalo is greater than that in cattle because this pathogen has evolved longer in the former host than in the later ${ }^{8}$. Similarly, in T. lestoquardi, the genetic diversity of the tams 1 homologous gene is lower than that of the tams1 gene because T. lestoquardi is thought to have recently evolved ${ }^{18}$. Therefore, there is a possibility that the new Theileria sp. was introduced in Sri Lanka relatively recently as a clonal population. If so, countries other than Sri Lanka may also be endemic to this Theileria sp. Epidemiological surveys to detect the new Theileria sp. in cattle from other countries may clarify this.

Previously, phylogenetic analyses conducted using $18 \mathrm{~S}$ rRNA sequences found that the transforming Theileria species, including T. parva, T. annulata, T. taurotragi, Theileria sp. (buffalo), and T. lestoquardi, have a common ancestor $^{20,25}$. Therefore, detecting the new Theileria sp. as a sister clade to the common ancestor of the T. annulata and T. lestoquardi sequences infers that the Theileria sp. may also be a transforming Theileria. However, isolation and in vitro cultivation of the schizont stage, together with genome sequencing analysis to identity the host cell transformation-related genes ${ }^{26}$, are essential to confirm whether the new Theileria sp. is a transforming species.

In conclusion, the present study, which aimed to analyse the genetic diversity of T. annulata in Sri Lanka, unexpectedly revealed a novel Theileria sp. However, the morphological, clinical, and pathological distinctions of this novel Theileria sp. are yet to be investigated. Therefore, the new species was provisionally designated as Theileria sp. Yokoyama.

\section{Methods}

DNA samples. Thirty-nine archived DNA samples from blood collected from cattle in Polonnaruwa $(\mathrm{n}=32)$ and Nuwara Eliya $(n=7)$ in June 2014 were used in the present study ${ }^{15}$. All animals were apparently healthy during sampling. DNA samples were prepared from $200 \mu$ l of whole blood using a commercial kit (Qiagen, Hilden, Germany) following the manufacturer's instructions. All DNA samples were positive for T. annulata via PCR assay based on the tams 1 gene ${ }^{15,27}$.

PCR amplification, cloning, and sequencing of tams1, tasp, 18S rRNA, and cob. The 18S rRNA and the tams 1, tasp, and cob gene sequences were amplified via PCR assays using primer sets designed in the present study. Briefly, three sets of forward and reverse primers were designed to amplify the full-length tams 1 , tasp, and $c o b$ genes using multiple alignments based on the T. annulata gene sequences retrieved from GenBank (Table 2). For the $18 \mathrm{~S}$ rRNA, T. annulata-specific forward and reverse primers were designed to amplify an 1,185-bp fragment based on multiple alignment of the 18S rRNA sequences from bovine Babesia and Theileria species, including B. bovis, B. bigemina, and T. orientalis, because some of the T. annulata-positive DNA samples were coinfected with these species ${ }^{15}$. In addition, previously described universal forward ${ }^{28}$ and reverse ${ }^{29}$ primers were used to amplify a long fragment of $18 \mathrm{~S}$ rRNA $(\approx 1600 \mathrm{bp})$ from nine DNA samples with single infection. Each PCR assay was conducted in a $10-\mu \mathrm{l}$ reaction mixture containing $1 \mu \mathrm{l}$ of T. annulata-positive genomic DNA, $1 \times$ PCR buffer $(10 \times$ PCR buffer, Applied Biosystems, Branchburg, NJ, USA), $200 \mu$ M of each dNTP (Applied Biosystems), $0.5 \mu \mathrm{M}$ of each forward and reverse primer, $0.1 \mu \mathrm{l}$ of $5 \mathrm{U} / \mu \mathrm{l}$ taq polymerase (Applied Biosystems), and $5.9 \mu \mathrm{l}$ distilled water. The reaction mixture was then subjected to an initial predenaturation step at $95^{\circ} \mathrm{C}$ for $5 \mathrm{~min}$, followed by $45 \mathrm{cycles}$ each of denaturation at $95^{\circ} \mathrm{C}$ for $30 \mathrm{sec}$, an annealing step at the appropriate temperature (listed in Table 1) for $30 \mathrm{sec}$, and an extension step at $72^{\circ} \mathrm{C}$ for $2 \mathrm{~min}$. After a final elongation step at $72^{\circ} \mathrm{C}$ for $7 \mathrm{~min}$, the PCR products were resolved by $1.5 \%$ agarose gel electrophoresis, stained with ethidium bromide, and visualized under ultraviolet (UV) illumination. PCR bands with appropriate sizes were gel-extracted, ligated to PCR 2.1 plasmid vectors, and sequenced using an ABI PRISM 3100 genetic analyser (Applied Biosystems). All sequences generated in the present study were trimmed to remove the primer regions before conducting the sequencing and phylogenetic analyses.

Gene sequences from GenBank. The T. annulata tams 1 gene sequences $(n=125)$ and coding sequences $(\mathrm{n}=14)$ of the tasp gene with $100 \%$ coverage to the Sri Lankan sequences were retrieved from GenBank. Similarly, 63 and 38 T. annulata 18S rRNA sequences with 100\% coverage to the Sri Lankan short and long 18S rRNA sequences, respectively, were also retrieved from GenBank. Nucleotide sequences with degenerate bases or an " $\mathrm{n}$ " symbol were omitted. In addition, some $18 \mathrm{~S}$ rRNA sequences registered as derived from T. annulata 
(MF287917, MF287919, MF287920, MF287924, MF287934, MF287937, MF287939, MF287942, MF287948, MF287949, MF287950, KT736499, KT367869, and KT367870) were ignored because they shared high identity scores with T. orientalis compared with T. annulata in our BLAST search. The GenBank-derived T. annulata nucleotide and amino acid sequences were aligned with the Sri Lankan sequences, and then trimmed to remove the excess nucleotides or amino acids. For the cob gene, the T. annulata sequences $(n=43)$ from GenBank with $98 \%$ coverage to the Sri Lankan sequences were obtained. These cob nucleotide sequences and translated amino acid sequences were aligned with the Sri Lankan sequences. Excess nucleotides or amino acids in the alignment were removed at the $3^{\prime}$ end or C-terminal based on the Sri Lankan sequences and at the $5^{\prime}$ end or N-terminal based on the Sudanese sequences.

Calculation of identity scores. Alignments of the $18 \mathrm{~S}$ rRNA sequences and nucleotide and amino acid sequences of tams1, tasp (coding region), and cob genes, which contained both the GenBank-derived T. annulata and Sri Lankan sequences, were analysed using MatGAT software ${ }^{30}$ to calculate the identity scores among the Sri Lankan sequences, among the GenBank-derived sequences, and between the Sri Lankan and GenBank-derived sequences.

Phylogenetic analyses. $18 \mathrm{~S}$ rRNA sequences and nucleotide and amino acid sequences of tams 1 , tasp (coding region), and $c o b$ genes from GenBank and those determined in the present study were subjected to multiple alignment using Multiple Alignment using Fast Fourier Transform (MAFFT) online software ${ }^{31}$. The aligned sequences were analysed using Molecular Evolutionary Genetics Analysis (MEGA), version 6.0 software ${ }^{32}$ to predict the best-fitting substitution models based on the lowest Akaike information criterion (AIC) values. Subsequently, using nucleotide sequences, five maximum likelihood phylogenetic trees were constructed based on general time reversible ${ }^{33}$ (tams1, tasp, and 18S rRNA-short) and Tamura-Nei ${ }^{34}$ (18S rRNA-long and cob) substitution models, using MEGA software ${ }^{32}$. Similarly, three maximum likelihood phylogenetic trees were constructed using amino acid sequences based on Dayhoff ${ }^{35}$ (TAMS1), Whelan And Goldman ${ }^{36}$ (TASP), and JTT ${ }^{37}$ (COB) substitution models. All positions containing gaps were removed from the alignments before phylogeny construction.

Ethical approval. The Animal Care and Use Committee of Obihiro University of Agriculture and Veterinary Medicine, Japan, approved all animal procedures (approval number: 29-53). All experiments were carried out in accordance with the Fundamental Guidelines for Proper Conduct of Animal Experiment and Related Activities in Academic Research Institutions coming under the Ministry of Education, Culture, Sports, Science and Technology, Japan.

\section{Data availability}

The materials and datasets generated in the present study are available from the corresponding author upon reasonable request.

Received: 8 April 2019; Accepted: 18 October 2019;

Published online: 06 November 2019

\section{References}

1. Bishop, R., Musoke, A., Morzaria, S., Gardner, M. \& Nene, V. Theileria: intracellular protozoan parasites of wild and domestic ruminants transmitted by ixodid ticks. Parasitology 129(Suppl.), S271-S283 (2004).

2. Robinson, P. M. Theileriosis annulata and its transmission-a review. Trop. Anim. Health Prod. 14, 3-12 (1982).

3. Brown, C. G. D. Theileriosis in Handbook on Animal Diseases in the Tropics (eds. Sewell, M. H. H. \& Brocklesby, D. W.) 183-199 (Balliere Tindall, 1990).

4. Gill, B. S., Bhattacharyulu, Y. \& Kaur, D. Symptoms and pathology of experimental bovine tropical theileriosis (Theileria annulata infection). Annales de Parasitologie 52, 597-608 (1977).

5. Brown, C. G. Dynamics and impact of tick-borne diseases of cattle. Trop. Anim. Health Prod. 29(4 Suppl.), 1S-3S (1997).

6. Inci, A. et al. Economical impact of tropical theileriosis in the Cappadocia region of Turkey. Parasitol. Res. 101(Suppl.), S171-S174 (2007).

7. Rashid, M. et al. Economic significance of tropical theileriosis on a holstein friesian dairy farm in Pakistan. J. Parasitol. 104, 310-312 (2018).

8. McKeever, D. J. Bovine immunity - a driver for diversity in Theileria parasites? Trends Parasitol. 25, 269-276 (2009).

9. MacHugh, N. D. et al. Extensive polymorphism and evidence of immune selection in a highly dominant antigen recognized by bovine CD8 T cells specific for Theileria annulata. Infect. Immun. 79, 2059-2069 (2011).

10. Katzer, F., McKellar, S., Ben Miled, L., D’Oliveira, C. \& Shiels, B. Selection for antigenic diversity of Tams1, the major merozoite antigen of Theileria annulata. Ann. N. Y. Acad. Sci. 849, 96-108 (1998).

11. Manuja, A. et al. Isolates of Theileria annulata collected from different parts of India show phenotypic and genetic diversity. Vet. Parasitol. 137, 242-252 (2006).

12. Schnittger, L. et al. Characterization of a polymorphic Theileria annulata surface protein (TaSP) closely related to PIM of Theileria parva: implications for use in diagnostic tests and subunit vaccines. Mol. Biochem. Parasitol. 120, 247-256 (2002).

13. Wang, J. et al. Genetic diversity and phylogenetic analysis of Tams1 of Theileria annulata isolates from three continents between 2000 and 2012. Cent. Eur. J. Immunol. 39, 476-484 (2014).

14. Sivakumar, T. et al. A PCR-based survey of selected Babesia and Theileria parasites in cattle in Sri Lanka. Vet. Parasitol. 190, 263-267 (2012).

15. Sivakumar, T. et al. A longitudinal study of Babesia and Theileria infections in cattle in Sri Lanka. Vet. Parasitol. Reg. Stud. Rep. 6, 20-27 (2016).

16. Gubbels, M. J., Katzer, F., Hide, G., Jongejan, F. \& Shiels, B. R. Generation of a mosaic pattern of diversity in the major merozoitepiroplasm surface antigen of Theileria annulata. Mol. Biochem. Parasitol. 110, 23-32 (2000).

17. Greay, T. L. et al. Endemic, exotic and novel apicomplexan parasites detected during a national study of ticks from companion animals in Australia. Parasit. Vectors 11, 197 (2018).

18. Al-Hamidhi, S. et al. Theileria lestoquardi displays reduced genetic diversity relative to sympatric Theileria annulata in Oman. Infect. Genet. Evol. 43, 297-306 (2016). 
19. Brown, C. G. et al. Theileria lestoquardi and T. annulata in cattle, sheep, and goats. In vitro and in vivo studies. Ann. N. Y. Acad. Sci. 849, 44-51 (1998).

20. Mans, B. J., Pienaar, R. \& Latif, A. A. A review of Theileria diagnostics and epidemiology. Int. J. Parasitol. Parasites Wildl. 4, 104-18 (2015).

21. Mans, B. J., Pienaar, R., Latif, A. A. \& Potgieter, F. T. Diversity in the 18 S SSU rRNA V4 hyper-variable region of Theileria in bovines and African buffalo (Syncerus caffer) from southern Africa. Parasitology 138, 766-779 (2011).

22. Mans, B. J., Pienaar, R., Potgieter, F. T. \& Latif, A. A. Theileria parva, T. sp. (buffalo) and T. sp. (bougasvlei) $18 \mathrm{~S}$ variants. Vet. Parasitol. 182, 382-383 (2011).

23. Chatanga, E. et al. Evidence of multiple point mutations in Theileria annulata cytochrome b gene incriminated in buparvaquone treatment failure. Acta Trop. 191, 128-132 (2019).

24. Mhadhbi, M., Chaouch, M., Ajroud, K., Darghouth, M. A. \& BenAbderrazak, S. Sequence polymorphism of cytochrome b gene in Theileria annulata Tunisian isolates and its association with buparvaquone treatment failure. PLoS One 10, e0129678 (2015).

25. Sivakumar, T., Hayashida, K., Sugimoto, C. \& Yokoyama, N. Evolution and genetic diversity of Theileria. Infect. Genet. Evol. 27, 250-263 (2014).

26. Hayashida, K. et al. Comparative genome analysis of three eukaryotic parasites with differing abilities to transform leukocytes reveals key mediators of Theileria-induced leukocyte transformation. MBio 3, e00204-e00212 (2012).

27. Kirvar, E. et al. Detection of Theileria annulata in cattle and vector ticks by PCR using the Tams1 gene sequences. Parasitology 120, 245-254 (2000).

28. Oosthuizen, M. C., Zweygarth, E., Collins, N. E., Troskie, M. \& Penzhorn, B. L. Identification of a novel Babesia sp. from sable antelope (Hippotragus niger, Harris 1838). J. Clin. Microbiol. 46, 2247-2251 (2008).

29. Matjila, P. T., Leisewitz, A. L., Oosthuizen, M. C., Jongejan, F. \& Penzhorn, B. L. Detection of a Theileria species in dogs in South Africa. Vet. Parasitol. 157, 34-40 (2008).

30. Campanella, J. J., Bitincka, L. \& Smalley, J. MatGAT: an application that generates similarity/identity matrices using protein or DNA sequences. BMC Bioinformatics 4, 29 (2003).

31. Katoh, K., Misawa, K., Kuma, K. \& Miyata, T. MAFFT: a novel method for rapid multiple sequence alignment based on fast Fourier transform. Nucleic Acids Res. 30, 3059-3066 (2002).

32. Tamura, K., Stecher, G., Peterson, D., Filipski, A. \& Kumar, S. MEGA6: molecular evolutionary genetics analysis version 6.0. Mol. Biol. Evol. 30, 2725-2729 (2013).

33. Nei, M. \& Kumar, S. Molecular evolution and phylogenetics. Oxford University Press, New York (2000).

34. Tamura, K. \& Nei, M. Estimation of the number of nucleotide substitutions in the control region of mitochondrial DNA in humans and chimpanzees. Mol. Biol. Evol. 10, 512-526 (1993).

35. Schwarz, R. \& Dayhoff M. Matrices for detecting distant relationships in Atlas of protein sequences (ed. Dayhoff, M.) 353-358 (National Biomedical Research Foundation, 1979).

36. Whelan, S. \& Goldman, N. A general empirical model of protein evolution derived from multiple protein families using a maximumlikelihood approach. Mol. Biol. Evol. 18, 691-699 (2001).

37. Jones, D. T., Taylor, W. R. \& Thornton, J. M. The rapid generation of mutation data matrices from protein sequences. Comput. Appl. Biosci. 8, 275-282 (1992).

\section{Acknowledgements}

We thank the staff at the Veterinary Research Institute, Sri Lanka, and Ms. Hiroko Yamamoto, Obihiro University of Agriculture and Veterinary Medicine, Japan, for their excellent technical assistance. This study was supported by grants from the Japan Society for Promotion of Science (JSPS) Grant-in-Aid for Scientific Research (JSPS KAKENHI Numbers 26257417 and 16H05033), Open Partnership Joint Projects of the JSPS Bilateral Joint Research Projects, and the AMED/JICA Science and Technology Research Partnership for Sustainable Development (SATREPS) project (Grant number 17jm0110006h0005).

\section{Author contributions}

T.S. and N.Y. conceived the study. T.S., S.F. and B.T. conducted the experiments and analysed the data. H.K. and S.S.P.S. conducted the cattle surveys and provided the laboratory facilities for the initial screening. T.S. and N.Y. wrote the manuscript, which all authors subsequently edited.

\section{Competing interests}

The authors declare no competing interests.

\section{Additional information}

Supplementary information is available for this paper at https://doi.org/10.1038/s41598-019-52512-y.

Correspondence and requests for materials should be addressed to N.Y.

Reprints and permissions information is available at www.nature.com/reprints.

Publisher's note Springer Nature remains neutral with regard to jurisdictional claims in published maps and institutional affiliations.

Open Access This article is licensed under a Creative Commons Attribution 4.0 International License, which permits use, sharing, adaptation, distribution and reproduction in any medium or format, as long as you give appropriate credit to the original author(s) and the source, provide a link to the Creative Commons license, and indicate if changes were made. The images or other third party material in this article are included in the article's Creative Commons license, unless indicated otherwise in a credit line to the material. If material is not included in the article's Creative Commons license and your intended use is not permitted by statutory regulation or exceeds the permitted use, you will need to obtain permission directly from the copyright holder. To view a copy of this license, visit http://creativecommons.org/licenses/by/4.0/.

(C) The Author(s) 2019 